Supporting Information to Accompany

\title{
New Peptidomimetic Polymers for Antifouling Surfaces
}

Andrea R. Statz, Robert J. Meagher ${ }^{\dagger}$, Annelise E. Barron ${ }^{\dagger}$, Phillip B. Messersmith ${ }^{*}$

Northwestern University, Department of Biomedical Engineering, 2145 Sheridan Road, Evanston, IL, 60208

\section{Complete Author List for Reference \#6:}

6. Simon, R. J.; Kania, R. S.; Zuckermann, R. N.; Huebner, V. D.; Jewell, D. A.; Banville, S.; Ng, S.; Wang, L.; Rosenberg, S.; Marlowe, C. K.; Spellmeyer, D. C.; Tan, R. Y.; Frankel, A. D.; Santi, D. V.; Cohen, F. E.; Bartlett, P. A. Proc. Natl. Acad. Sci. U. S. A. 1992, 89, 9367-9371.

\section{EXPERIMENTAL PROCEDURES and CHARACTERIZATION DATA}

\section{Polymer Synthesis and Purification}

PMP1 was synthesized on Rink amide resin (Nova Biochem, San Diego, CA) using an ABI 433A (Applied Biosystems, Foster City, CA) automated peptide synthesizer. Conventional Fmoc strategy of solid phase peptide synthesis with Fmoc-Lys-(N-Boc) and Fmoc-DOPA(acetonid) amino acids (NovaBiochem, San Diego, CA) was used to synthesize the C-terminal DOPA-Lys-DOPA-Lys-DOPA peptide anchor, after which the polypeptoid portion was synthesized using a submonomer protocol described previously. ${ }^{1}$ Following completion of the synthesis, the $N$-terminus of the peptidomimetic polymer was acetylated with acetic anhydride (Applied Biosystems, Foster City, CA). Cleavage of the peptidomimetic polymer from the resin and deprotection of the amino acid side chains was accomplished by treating the resin with 95\% (v/v) trifluoroacetic acid (Acros Organics, Belgium) with $2.5 \% \mathrm{H}_{2} \mathrm{O}$ and $2.5 \%$ triisopropylsilane (Aldrich, Milwaukee, WI). The cleaved polymer was isolated by

\footnotetext{
${ }^{\dagger}$ Department of Chemical and Biological Engineering
} 
filtration and rinsed several times with acetonitrile and water. The crude product was analyzed by reversed-phase HPLC using a Vydac C18 column and MALDI-MS for purity and composition. Purification was performed by preparative HPLC, and purified fractions were frozen at $-85^{\circ} \mathrm{C}$ and lyophilized.

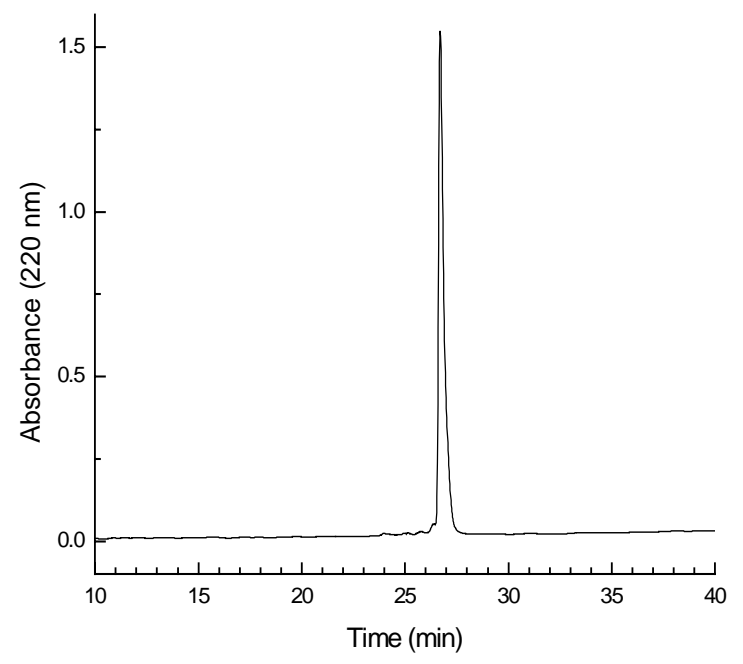

Figure S1. Analytical RP-HPLC of purified PMP1 (Vydac C18, 10-60\% Acetonitrile in $\mathrm{H}_{2} 0$ with $0.1 \%$ $\mathrm{v} / \mathrm{v}$ TFA, $0.5 \mathrm{ml} / \mathrm{min}, 50$ minutes).

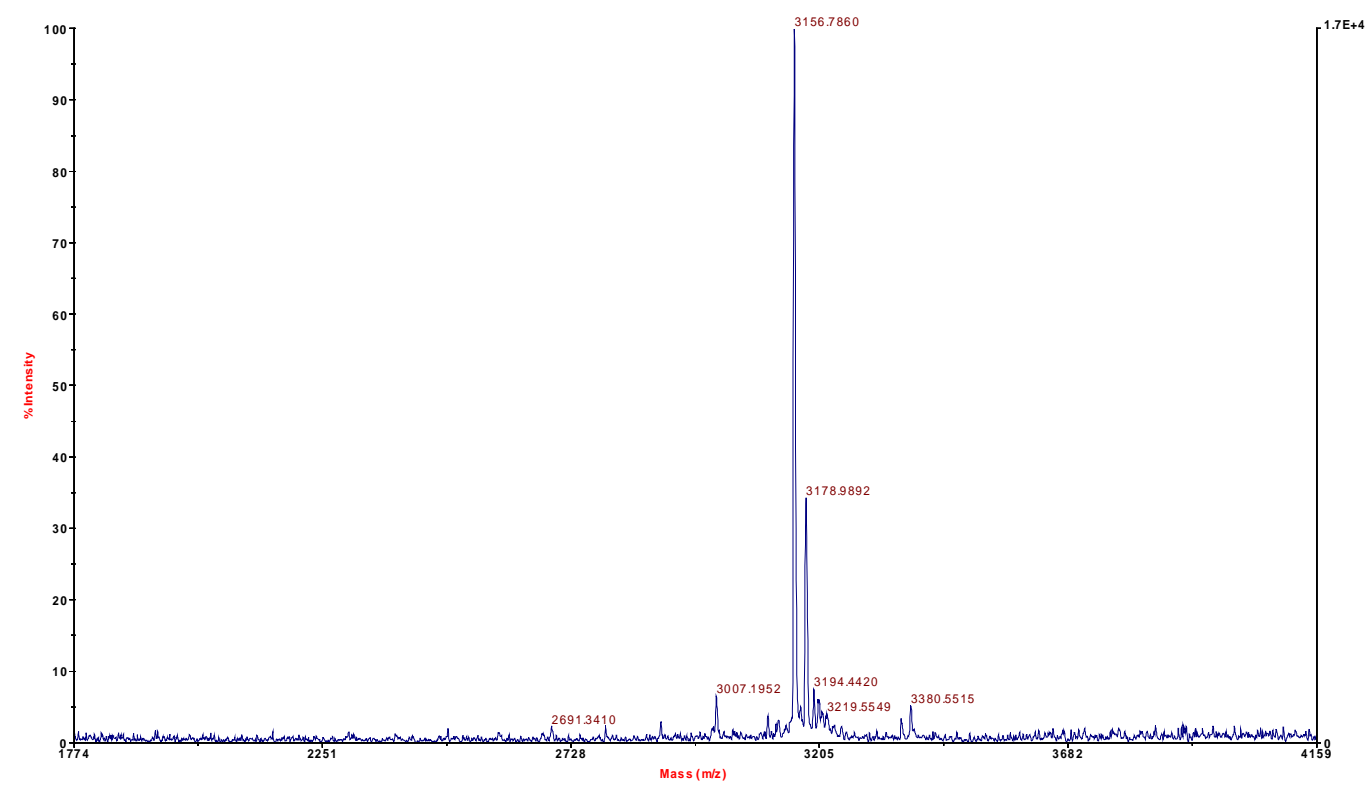

Figure S2. MALDI-MS spectrum of purified PMP1. Determined molecular weight $\mathrm{m} / \mathrm{z} 3156.7860$ (calculated molecular weight 3153.67). 


\section{Substrate Preparation}

Silicon wafers were coated with a $20 \mathrm{~nm}$ thick layer of Ti by electron beam-evaporation and then cut into $8 \mathrm{~mm}$ by $8 \mathrm{~mm}$ pieces. The substrates were cleaned ultrasonically for ten minutes in 2-propanol, dried under $\mathrm{N}_{2}$, and then exposed to $\mathrm{O}_{2}$ plasma (Harrick Scientific, Ossining, USA) at $\leq 150$ Torr and $100 \mathrm{~W}$ for three minutes to produce a clean titanium oxide surface. Under the aqueous conditions of the adsorption experiments used in preparation of the modified surfaces, the nature of the Ti surface is expected to be an oxide of approximate composition $\mathrm{TiO}_{2} \cdot{ }^{2}$ OWLS waveguides were purchased from Microvacuum Ltd. (Budapest, Hungary), consisting of a $\mathrm{SiO}_{2}$ substrate coated with $\mathrm{Si}_{0.25} \mathrm{Ti}_{0.75} \mathrm{O}_{2}$ and a final $10 \mathrm{~nm}$ thick coating of $\mathrm{TiO}_{2}$ produced by a sol-gel process. ${ }^{3}$ Sensors were cleaned following the same procedure as for Ti substrates.

\section{Substrate Modification}

Clean substrates were immersed in $1 \mathrm{mg} / \mathrm{ml}$ PMP1 in saturated $\mathrm{NaCl}$ buffered with $0.1 \mathrm{M} N$ morpholinopropanesulfonic acid (MOPS) at $60^{\circ} \mathrm{C}$ for 24 hours. OWLS waveguides were similarly modified using a $1 \mathrm{mg} / \mathrm{ml}$ solution of PMP1 in ultrapure $\mathrm{H}_{2} \mathrm{O}$. After modification, substrates and waveguides were exhaustively rinsed with ultrapure $\mathrm{H}_{2} \mathrm{O}$ and dried in a stream of filtered $\mathrm{N}_{2}$.

\section{Surface Characterization}

Survey and high resolution XPS spectra were collected on an Omicron ESCALAB (Omicron, Taunusstein, Germany) configured with a monochromated Al Ko (1486.8 eV) 300-W X-ray source, 1.5 mm circular spot size, a flood gun to counter charging effects, and an ultrahigh vacuum $\left(<10^{-8}\right.$ Torr $)$. The takeoff angle, defined as the angle between the substrate normal and the detector, was fixed at $45^{\circ}$. Substrates were mounted on standard sample studs by means of double-sided adhesive tape. All binding energies were calibrated using the $\mathrm{C}(1 \mathrm{~s})$ carbon peak $(284.6 \mathrm{eV})$. Analysis consisted of a broad survey scan (50.0 eV pass energy) and a 10-min high-resolution scan (22.0 eV pass energy) at 275-295 $\mathrm{eV}$ for $\mathrm{C}(1 \mathrm{~s})$ and $390-410 \mathrm{eV}$ for $\mathrm{N}(1 \mathrm{~s})$. 
High-resolution spectra were acquired and used to calculate atomic composition (Table 1), showing a significant increase in carbon and nitrogen and a significant decrease in titanium and oxygen upon adsorption of PMP1 onto the Ti surface. These spectra showed an increase in the ether $(\mathrm{C}-\mathrm{O})$ peak at $286.0 \mathrm{eV}$ when compared to control Ti surfaces (Figure S3). A peak at $284.6 \mathrm{eV}$ is due to the aliphatic and aromatic carbons in the methoxyethyl side chain and DOPA anchoring group, as well as to some hydrocarbon contamination. The carbonyl groups of the peptidomimetic polymer backbone are represented by a peak at $287.5 \mathrm{eV}$. Furthermore, a strong N(1s) peak was present at $399.7 \mathrm{eV}$ in the spectra of the PMP1-modified surfaces, that was not observed in the spectra of the unmodified Ti surfaces (Figure S3).

Table S1. Atomic composition of unmodified and PMP1 modified Ti as determined by XPS.

\begin{tabular}{|l|l|l|l|l|}
\hline & $\% \mathrm{Ti}$ & $\% \mathrm{O}$ & $\% \mathrm{C}$ & $\% \mathrm{~N}$ \\
\hline Unmodified Ti & 22.8 & 62.4 & 14.8 & 0.0 \\
\hline PMP1 Modified Ti & 2.98 & 21.0 & 55.6 & 20.4 \\
\hline PMP1 Theoretical & & 28.5 & 58.0 & 13.4 \\
\hline
\end{tabular}

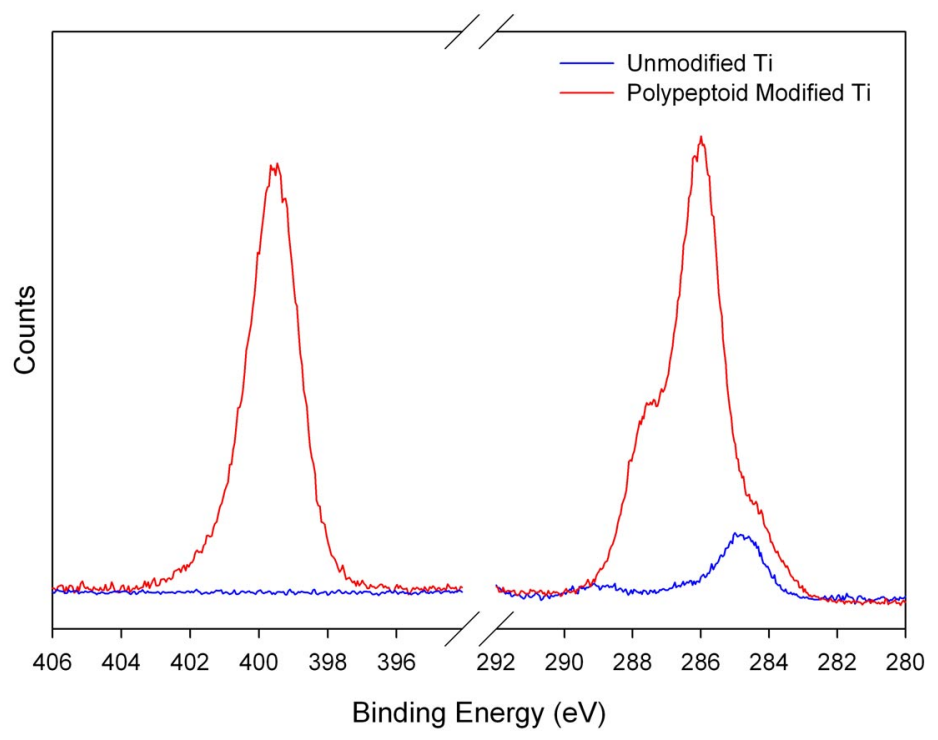


Figure S3. XPS analysis indicates the presence of adsorbed PMP1 on modified Ti surfaces. Highresolution N(1s) (left) and C(1s) (right) XPS spectra of unmodified and PMP1-modified Ti substrates.

Secondary ion spectra were recorded on a TRIFT III time-of-flight secondary ion mass spectrometer (Physical Electronics, Eden Prairie, MN) in the mass range 0-2000 m/z. A Ga ${ }^{+}$-source was used at a beam energy of $15 \mathrm{keV}$ with a $100 \mu \mathrm{m}$ raster size. Positive and negative spectra were collected and calibrated with a set of low mass ions using the PHI software Cadence.

The positive ion ToF-SIMS spectrum of unmodified Ti exhibited typical low-intensity hydrocarbon contamination peaks $\left(\mathrm{C}_{\mathrm{n}} \mathrm{H}_{2 \mathrm{n}+1}{ }^{+}\right.$and $\left.\mathrm{C}_{\mathrm{n}} \mathrm{H}_{2 \mathrm{n}-1}{ }^{+}\right)$, a Ti peak at $m / z=47.95$ and a $\mathrm{TiO}^{+}$peak at $m / z=63.95$. The positive ion ToF-SIMS spectrum of PMP1-modified Ti revealed numerous fragments representing the presence of adsorbed PMP1 (Figure S4). Apparent fragmentation of the methoxyethyl side chain of the peptoid repeat gave rise to peaks for $\mathrm{C}_{2} \mathrm{H}_{5} \mathrm{O}^{+}(m / z=45.06), \mathrm{C}_{3} \mathrm{H}_{7} \mathrm{O}^{+}(m / z=59.07)$ and $\mathrm{C}_{4} \mathrm{H}_{10} \mathrm{NO}^{+}$ $(m / z=88.11)$ fragments, and also Lys-derived fragments such as $\mathrm{C}_{2} \mathrm{NH}_{4}{ }^{+}(\mathrm{m} / \mathrm{z}=42.04)$. The negative ion spectrum contained peaks for 1-, 2-, and 3-mer peptoid fragments as well as other large fragments of the peptoid portion of PMP1 (Figure S5). Additionally, fragments corresponding to Ti complexed with the catechol, DOPA and peptoid residues are also visible in the negative ion spectrum (Figure S5).

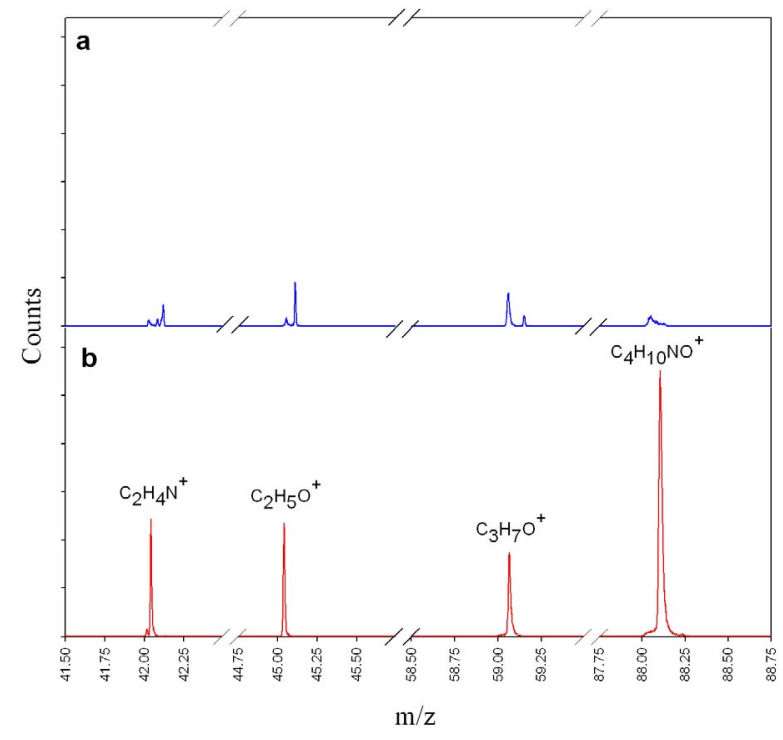


Figure S4. The low-mass region of the positive ion ToF-SIMS spectra of (a) unmodified Ti and (b) Ti modified with PMP1.

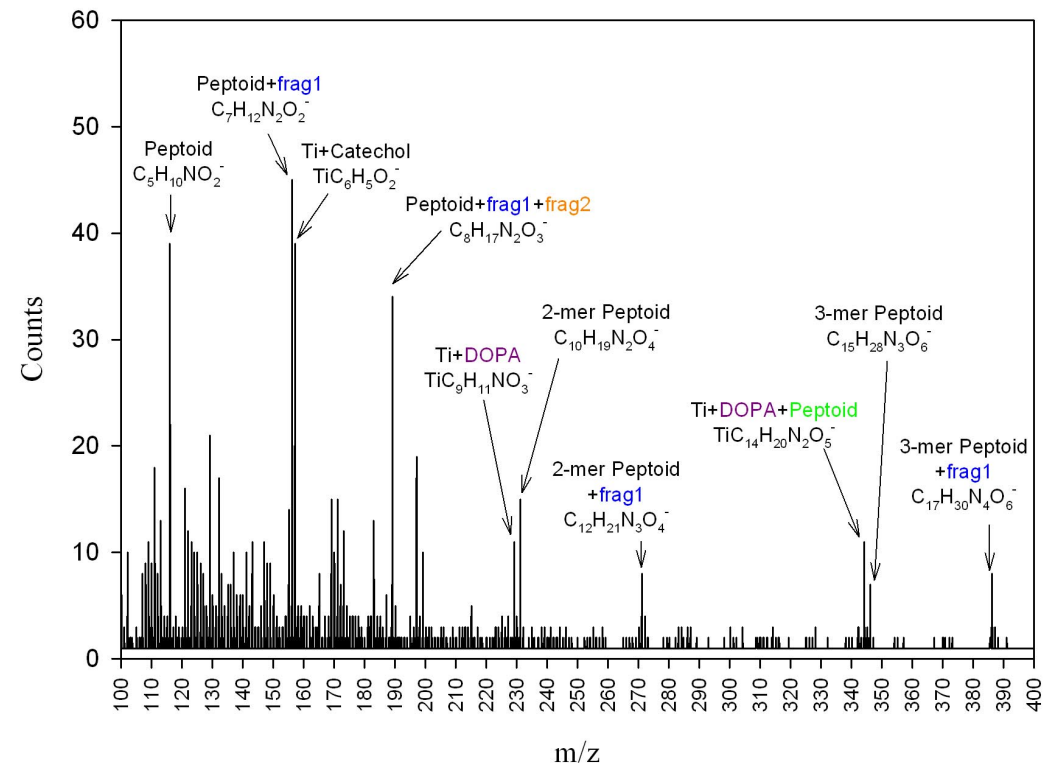

Figure S5. The mid-range mass region of the negative ion ToF-SIMS spectrum of Ti modified with PMP1.

\section{Protein Adsorption}

OWLS experiments were performed as described in Dalsin et al. ${ }^{4}$ and Voros et al. ${ }^{3}$ For in situ protein adsorption experiments, $\mathrm{TiO}_{2}$ coated waveguides were modified ex situ with PMP1. The waveguides were inserted in the measurement head of an OWLS110 (Microvacuum Ltd.) and equilibrated by exposure to HEPES buffer (10 mM HEPES, $150 \mathrm{mM} \mathrm{NaCl}, \mathrm{pH} 7.4)$ through the flow-through cell for at least 6 hours, to allow for complete exchange of ions at the $\mathrm{TiO}_{2}$ surface. The measurement head was mounted in the sample chamber and heated to $37^{\circ} \mathrm{C}$; the signal was recorded to ensure a stable baseline and thus adequate equilibration time. Whole human serum (Control Serum N, Roche Diagnostics, Switzerland, reconstituted in ultrapure water) was injected into the flow-through cell in stop-flow mode. The waveguide was exposed to serum for 20 minutes and subsequently rinsed with HEPES buffer for 
another 40 minutes. The measured incoupling angles, $\alpha_{\mathrm{TM}}$ and $\alpha_{\mathrm{TE}}$ were converted to refractive indices, $N_{\mathrm{TM}}$ and $N_{\mathrm{TE}}$ by the Microvacuum software, and changes in refractive index at the sensor surface were converted to adsorbed mass using de Feijter's formula. ${ }^{4}$

The refractive indices of solutions were measured in a refractometer (J157 Automatic Refractometer, Rudolph Research) under identical experimental conditions. A refractive index value of $1.33119 \mathrm{~cm}^{3} / \mathrm{g}$ was used for the HEPES buffer and a standard value of $0.182 \mathrm{~cm}^{3} / \mathrm{g}$ was used for the protein-adsorption calculations. The large mass increase upon injection of serum reflects protein adsorption as well as the change in refractive index of the covering solution near the surface. The residual increase in signal intensity versus baseline measured by OWLS can be directly correlated to adsorbed mass of protein. ${ }^{3}$

\section{Cell Adhesion Experiment}

3T3-Swiss albino fibroblasts (ATCC, Manassas, VA) were maintained at $37^{\circ} \mathrm{C}$ and $5 \% \mathrm{CO}_{2}$ in Dulbecco's modified Eagle's medium (DMEM, Cellgro, Herndon, VA) containing 10\% fetal bovine serum (FBS) and $100 \mu \mathrm{g} / \mathrm{ml}$ of penicillin and $100 \mathrm{U} / \mathrm{ml}$ of streptomycin. Trypsinized cells (passage 1216) were resuspended in DMEM with 10\% FBS and counted using a hemacytometer.

Modified and unmodified Ti substrates were pretreated in a 12-well TCPS plate with $1.0 \mathrm{ml}$ of DMEM/FBS for 30 minutes after which fibroblasts were seeded onto the test substrates at a density of $2.9 \times 10^{3}$ cells $/ \mathrm{cm}^{2}$. Cell culture medium was replenished on a regular basis and substrates were reseeded with $3 \mathrm{~T} 3$ fibroblasts at a density of $2.9 \times 10^{3}$ cells $/ \mathrm{cm}^{2}$ twice per week throughout the course of the experiment. Fibroblasts were stained with $2.5 \mu \mathrm{M}$ calcein-AM (Molecular Probes) in complete PBS for 1 hour at $37^{\circ} \mathrm{C}$ twice per week initially and then once per week after 2 weeks.

At each time point the medium was aspirated from each well to remove any non-adherent cells, and the substrates and wells were rinsed with PBS. Quantitative cell attachment data was obtained by acquiring nine images from random locations on each substrate using an Olympus BX-40 $\left(\lambda_{\mathrm{Ex}}=\right.$ 549nm, $\lambda_{\mathrm{Em}}=565 \mathrm{~nm}$ ) and a Coolsnap CCD camera (Roper Scientific, Trenton, NJ). The experiments were performed in triplicate for statistical purposes, resulting in a total of 27 images per time point for 
each substrate. The resulting images were quantified using thresholding in Metamorph (Universal Imaging, Downington, PA).

\section{References}

(1) Zuckermann, R. N.; Kerr, J. M.; Kent, S. B. H.; Moos, W. H. J. Am. Chem. Soc. 1992, 114, 10646-10647.

(2) Brunette, D. M.; Tengvall, P.; Textor, M.; Thomsen, P., Eds. Titanium in Medicine; SpringerVerlag: Berlin, 2001.

(3) Voros, J.; Ramsden, J. J.; Csucs, G.; Szendro, I.; De Paul, S. M.; Textor, M.; Spencer, N. D. Biomaterials 2002, 23, 3699-3710.

(4) Dalsin, J.; Tosatti, S.; Vörös, J.; Textor, M.; Messersmith, P. B. Langmuir 2005, 21, 640-646. 